\title{
Navigation Assistance in Virtual Worlds
}

\section{Betsy van Dijk, Rieks op den Akker, Anton Nijholt, and Job Zwiers University of Twente, The Netherlands}

\author{
DvdikK@s.utwente.n
}

\begin{abstract}
In this paper we report about ongoing research on navigation assistance in virtual environments. Our aim is to contribute to the development of forms of navigation assistance that enable non-professional visitors of a virtual environment to find their way without previous training. The environment used in this research is a virtual theatre that models a real world music theatre. This virtual theatre can be used for exploration as well as for transactions and goal-directed search for information. We first present some design principles for navigation assistance in virtual environments and some design criteria for assistance by personal agents. Subsequently we describe how these principles and criteria have been implemented in our experimental virtual theatre environment. Finally we give an overview of future research plans.
\end{abstract}

Keywords: Navigation assistance, Personal agents, Virtual environments

\section{Introduction}

Non-professional users have often been observed to experience great difficulties when navigating in a virtual environment. In this paper we report on work in progress that focuses on navigation assistance for visitors of such an environment, the virtual theatre. This virtual theatre, a virtual environment that models an existing music theatre, has been built using VRML (Virtual Reality Modeling Language) and can be accessed through the World Wide Web. Thus far, the virtual theatre has been used as a laboratory for research, in particular for experiments with users interacting with agents in multimodal ways (using modalities like speech, natural language, mouse clicks, keyboard, and gaze).

Nijholt (2000a) reports about the e-commerce aspects of this environment and in Nijholt (2000b) attempts to let this environment evolve into a theatre community are discussed. In this community, goal-directed visitors can get information about performances and they will be able to make reservations for these performances (buy tickets).

Material published as part of this proceedings, either on-line or in print, is copyrighted by the author with permission granted to the publisher of Informing Science for this printing. Permission to make digital or paper copy of part or all of these works for personal or classroom use is granted without fee provided that the copies are not made or distributed for profit or commercial advantage AND that copies 1) bear this notice in full and 2) give the full citation on the first page. It is permissible to abstract these works so long as credit is given. To copy in all other cases or to republish or to post on a server or to redistribute to lists requires specific permission from the author.
Equally important is that visitors who have no specific information demands and who are not sure if they want to buy a ticket can just have a look around and explore the environment. Hence the environment is not only a goaldirected system for information and transaction but also an environment for exploration and entertainment.

These two types of activity - goal-directed and explorative - imply different types of navigation, hence they call for different types of navigation assistance. In this paper various forms of navigation assistance will be discussed. Some forms may be particularly useful in the case of goaldirected navigation whereas other forms are more likely to support explorative navigation. What all these forms have in common is that they should enable visitors of the virtual theatre to navigate in this environment, which includes visitors who have no training in navigation in virtual environments and who are not familiar with the environment. Navigation in general will be discussed in the next section, and navigation assistance in the third section. Then a specific form of assistance is presented in greater detail, assistance by personal agents. Such agents are dynamic, offering assistance that will always be based on the most recent information concerning the virtual environment. Another distinguishing feature of personal agents is the ability, or at least the potential ability, to offer assistance that complies with some known characteristics of a specific visitor. We describe three research projects of ours where personal agents and other forms of navigation assis- 


\section{Van Dijk, Op den Akker, Nijholt, Zwiers}

tance have been implemented. Finally in the last section we conclude with an overview of future research plans.

\section{Navigation}

Navigation originally was defined as the process of moving through an environment. Darken \& Sibert (1993) extend this definition of navigation to include the process of wayfinding (determining a path to be traveled). They define navigation as the process of determining and traveling a path through an environment. They extend this definition even more with a reference to the aids and cues people need for successful navigation in virtual environments, to arrive at the following definition: "Navigation is the process by which people control their movement using environmental cues and artificial aids such as maps so that they can achieve their goals without getting lost" (Darken \& Sibert, 1993, p157).

The World Wide Web allows the presentation through webpages of explicitly predefined information in the form of text, tables, pictures, audio, video and animation. Whereas the navigation activities on traditional twodimensional web-pages mainly comprise searching for information, with the development of virtual worlds the attention for exploration currently increases. Darken \& Sibert (1996) present a classification of wayfinding tasks in which they distinguish exploration (defined as a wayfinding task without a target) from search activities, where search activities are subdivided in naive search (the navigator has no a priori knowledge of the whereabouts of the target) and primed search (the navigator knows the location of the target). Benyon \& Höök (1997) also categorize navigation activities to be either goal-oriented (how to find a way to reach a known destination), or explorative (just interested to have a look around and find out what's there), or aiming at object identification (finding categories and clusters of objects spread across the environment, finding interesting configurations of objects, finding information about the objects). It is useful to add that these categories, though mutually exclusive (Darken \& Sibert, 1996), may become connected during navigation: exploration may invoke goal-oriented search activities, but the opposite direction is conceivable as well.

It has often been noted that navigation in virtual environments is difficult. Unfamiliar environments, whether virtual or real, are always difficult to navigate (Vinson, 1999), but in virtual environments more problems may be expected. Problems associated with wayfinding in virtual worlds may occur, first of all, because virtual environ- ments in general present less sensory (visual, auditory, locomotive) detail than real world environments. As a consequence, virtual environments contain fewer spatial and locomotive cues than real environments (Vinson, 1999). What adds to the complexity of wayfinding in virtual worlds is the fact that virtual environments tend to change faster than real world environments, hence for users it will be more difficult to become familiar with such environments. As a further point, the concept of navigation in hyperspace may have completely different physics from navigation in the physical world: movements can be made in virtual environments that have no counterpart in real world environments. For instance node-link representations in hypermedia and on the web permit discrete movement while movement in Euclidean space is continuous (Spence, 1999). Input by keyboard and mouse allows the user to move and to rotate, to jump from one location to another, to interact with objects and to trigger them. The user also has the possibility to view the world from different perspectives (Satalich, 1995). As a consequence, visitors of a virtual world often will encounter situations where they will not be able to rely on navigation skills acquired in the real world, as familiar kinds of cues are inefficient or inappropriate. Navigation problems like disorientation, loss of overview, difficulty to return to a location visited before or to refind an object found before, can lead to dissatisfaction, frustration and eventually discontinued use of that environment (Nash et al., 2000).

The process of determining a path (wayfinding) is inherently cognitive in nature (Nash et al., 2000). Many studies of navigation focus on understanding the knowledge and abilities it requires (e.g. Krieg-Brückner et al., 1998; Werner et al. 2000; Spence, 1999; Neerincx \& Lindenberg, 1999). These studies often compare real world wayfinding to wayfinding in virtual worlds. Theories on spatial knowledge and navigational awareness seem to be relevant for wayfinding in both the physical world and virtual worlds. In spatial knowledge theory two types of knowledge are distinguished:

- $\quad$ survey knowledge is characterized as the ability to conceptualize the space as a whole. This knowledge is map-like in nature: many routes and landmarks are combined into a cognitive map of the environment.

- procedural or route knowledge is defined as the sequence of actions required to follow a particular route. A person who has only procedural knowledge of a route can go from one landmark to another on that route but does not recognize alternative routes. 


\section{Navigation Assistance in Virtual Worlds}

In the literature it is agreed upon that survey knowledge is the key to successful effective navigation (e.g. Darken \& Sibert, 1996; Nash et al.,2000). To be able to create a cognitive map of the virtual environment, a user should be able to orient him/herself in space and build up landmarks, route and survey knowledge (Volbracht \& Domik, 2000). Survey knowledge can best be attained by exploration. Part of the knowledge can be attained through map or picture study alone, but orientation skills and the ability to determine one's position in the virtual environment require exploration as a means to acquire personal experience. Navigators with complete survey knowledge are said to possess 'navigational awareness'. In the U-WISH project, to be described in some detail further on, the related concept 'situational awareness' is adopted. This concept often is used to study task performance in domains like aviation or process control that are complex because they change continuously. Situational awareness is defined as a person's momentary knowledge of the surroundings and his/her presence in it (Neerincx et al., 2000). The main difference between the definitions of navigational and situational awareness is the term momentary, which refers to the fact that web-based and virtual environments change continuously.

\section{Navigation Assistance}

As navigation in virtual worlds is evidently difficult and as many virtual environments have been designed to be used by untrained visitors that explore the environment, navigation support is needed. Currently, much research on navigation and wayfinding is being conducted that seeks to develop applications for easing the task of navigating in a virtual environment. Based on the role of spatial knowledge in real world wayfinding tasks, Darken and Sibert (1996) suggest that principles for real world wayfinding can be used for constructing aids for wayfinding in virtual environments. They present a set of design principles for wayfinding support to virtual worlds. Some of these design principles concern the organisation of the environment, other design principles propose the addition of map-like information to the environment. With respect to the organisation, Darken and Sibert advise to divide large-scale worlds into distinct parts that are simply organized. Thus, they suggest, structure is provided that will enable the users to mentally organize the environment. Directional cues and visual and auditory cues can be added to the environment in order to ease recognition by the user of these parts and subparts and their organisation. The principles concerned with the addition of map-like information are intended to present spatial information directly in such a way that the user can produce a flexible orientationindependent representation of the environment. The basic principles are to show paths, landmarks, subparts and their organisation and the user's position. Furthermore Darken and Sibert advise to orient the map towards the user such that "the forward-up equivalence principle is accomodated": the map is presented in the same orientation as the environment itself so that for users it feels as if the map was in front of their chest. All these aids may help users to explore the virtual environment, enabling them to build a cognitive map of the environment and to develop 'situational awareness'.

In their study of real world-derived assistance, however, Darken and Sibert do not discuss personal assistents like guides and mentors. In virtual as well as real world environments, such personal assistents can be very useful aids, helping people to find their way or to find relevant information. In virtual environments personal assistants can give advice or make suggestions about interesting places to go to, how to get there, where to find or buy things or how to solve certain problems. They can guide the user (route guidance/guidance in problem solving) or transport the user directly, in which case the user hands over control to the assistent.

Personal assistants - or, as we call them, personal agents are already operational in our virtual theatre environment, for experimental purposes, and their presence and functioning is subject of continuing adjustment and elaboration. In the next section we will describe these personal agents in general, and their specific appearances and tasks.

\section{Personal Agents}

Personal agents for assisting wayfinding in virtual environments have many characteristics in common with agents for assisting wayfinding in real world environments, but there are clear differences as well. Some of these differences derive from the fact that virtual environments pose fewer restrictions on the number, the shaping, or even the knowledge of personal assistants than do real world environments. Other differences, in contrast, stem from virtual agents' rather limited abilities to combine and integrate a variety of different tasks, to cooperate with 'fellow agents', and to communicate with users across the boundaries of their own task domain. Thus, in some respects virtual environments may offer more wayfinding assistance than real world environments, but in other respects less assistance can be provided. For users this may be confus- 


\section{Van Dijk, Op den Akker, Nijholt, Zwiers}

ing, or even frustrating. The large amounts of ready knowledge of virtual agents, and the high quality graphical displays to convey this knowledge may set very high user expectations that cannot be met in subsequent agent-user communication, nor in subsequent agent-agent cooperation.

In the present section we will discuss the appearance and the knowledge base(s) of personal assistents in virtual environments, presenting some important design criteria for such agents. In the section to follow we will present the way the organisational and map design principles and personal agents actually feature in our virtual theatre. In the final section we will discuss our plans for future research on navigation assistance, emphasizing personal agents; we will discuss both technical improvements and their implications for the interaction between human and computer actors.

A first design criterion relates to the agent's visual (and perhaps auditive) appearance: an agent may be embodied or not. An embodied agent will tend to be more obtrusive (or less unobtrusive) than a non-embodied agent. When embodied, its appearance may be a cartoon personality, a human-like personality, or another character. Whatever the character that is decided upon, additional choices may concern the agent's facial expression (possibly changing), the agent's response to a user's gaze, that is, watching direction. It is conceivable to make the agent's appearance dependent on user characteristics (e.g. the user's age).

A second criterion relates to the agent's behavior: proactive or reactive. A pro-active agent may address a user without being asked for that by the user, while a reactive agent has to be deliberately activated by the user, for instance by means of a question or a mouse click. Note that this is a distinction that refers to user perceptions. From a technical viewpoint nearly all agents will be reactive, acting either in response to a question or to some observed user behavior. For example, an agent that offers guidance when a user seems to 'walk in circles' is acting reactively from a technical point of view, but a user will not consider this behavior as reactive, probably. To be complete, it is added here that agents can be designed to be pro-active as well as reactive, addressing users without being asked for that but also reacting when users ask for assistance.

Whereas these two criteria concerned agent characteristics that users may readily observe, a third criterion can be added that pertains to a characteristic less obvious for users: the agent's adaptiveness, which we define as the agent's ability to include knowledge about user-related changes in the assistance offered to that particular user. An adaptive agent is a dynamic agent, that is, an agent whose behavior is guided by an evolving knowledge base. However, agents can be dynamic without being adaptive. To provide assistance, a agent must have adequate, up-todate knowledge of the virtual environment. This environment will change constantly. In the virtual theatre, for instance, new performances are added to the schedule, and yesterday's performances are removed from the schedule. Moreover, performances can be fully-booked, they can be cancelled, or the starting time can be changed. A virtual agent whose task is to provide reservation assistance needs to have the most recent information about the performances schedule. This agent is dynamic, but not adaptive in the sense of applying user-related information. We do not present this purely environment-related agent dynamics as a design criterion, as we consider the ability to provide correct information a necessary property of any agent.

On the other hand, not every agent that applies user-related information is dynamical. If an agent takes user information into account that was collected at some point in time - for instance, at the occasion of the user's first visit or registration - and that information is not a subject of later verification, revision, or extension, there is no user-related dynamics. In that case too, we choose not to consider the agent as adaptive but rather use the term personalized agent.

To be able to offer user-specific assistance, an adaptive agent will have to make use of information that relates to a particular user. It is clear that in many situations we can expect different user interaction behaviors and different user preferences with respect to the content that is offered. These differences follow from different interests, background, culture, intelligence and interaction capabilities of users. These issues can become part of a user profile and will be used by the agents to anticipate the users preferences or to guide a user in reaching a particular place of the user's choice. This user profile may be concise or detailed, it may include relatively stable user characteristics (e.g. age, family composition) and/or more variable or less certain characteristics (e.g. preference categories). It may be based on information directly provided by the user (for instance by means of fill-in forms) and/or on information gathered from the user's previous reservations and 'physical' movements in the virtual environmen, the present position, the time spent to read about (or watch previews of) particular performances, and/or the user's responses to 


\section{Navigation Assistance in Virtual Worlds}

previous suggestions made by the assistant. Which information is needed for a particular agent depents on the tasks of that agent.

Already from this broad sketch of design criteria it may be clear that a wide variety of virtual agents is possible, ranging from non-embodied, silent, reactive, non-adaptive agents (that may resemble the help-menus as provided by many programs) to embodied, pro-active, adaptive virtual agents that may provide verbal assistance. How a particular agent is to be designed may depend on various considerations, e.g. the tasks to be performed by this agent, the characteristics of other agents in the virtual environment, assumptions about what users need or prefer, and assumptions about the degree of complexity users are able to manage. In the next section we will describe the virtual agents as they have been designed for the purpose of studying wayfinding assistance in the virtual theatre. As our research is about wayfinding assistance in general, not merely wayfinding assistance as provided by virtual agents, we will also discuss the assistance provided by means of landmarks and maps.

\section{Navigation Assistance in the Virtual Theatre Environment}

In the virtual theatre environment several of the navigation assistance principles have been implemented for the sake of experimentation and research. Presently this research takes place in different research projects, each emphasizing different aspects of navigation assistance. In this section we successively discuss the information and transaction agent, the agent and other support functions studied in the U-WISH project, and the natural language navigation agent. In the future we hope to combine the results of these research projects on navigation assistance in the design of one virtual environment with several specialized personal navigation agents, each with their own tasks, that interact with each other and with the user.

\section{The information and transaction agent}

The first personal agent to be mentioned is Karin, the receptionist of the virtual theatre. Karin is an information agent that sits behind an information desk near the entrance of the building. She gives information about the performances that are scheduled in the local theatres of our home town (dates, prices, available places, information about the artists and the performances), and she can make reservations (sell tickets). Hence Karin doesn't support navigation in the virtual world but navigation in the data- base that contains information about all forthcoming performances.

Karin is an embodied agent (see figure 1) with a talking 3D cartoon face with simple facial expressions. Lip movements are synchronized with a text-to-speech system that mouths Karin's responses to the user.

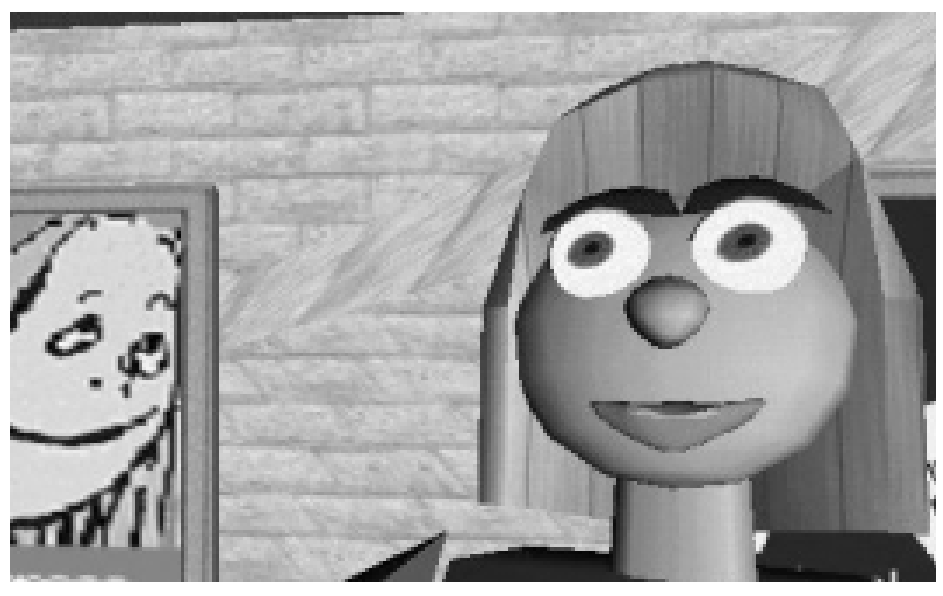

Figure 1. Karin, the virtual receptionist in our virtual theatre

This agent is reactive and only answers to questions of visitors. Interaction between Karin and the visitors are multimodal: the questions can be posed in natural language either by typing or by speech and the answers are not only synthesized but can be displayed in a special menu window in the form of written text (a table if many alternatives are available). Presently this information agent is not adaptive yet and she doesn't have notice of her visual environment. Hence deictic references of users to that visual context will not be understood and the context will not be helpful in interpreting and recognizing user utterances. In future research Karin will be made aware of her surroundings. Knowledge of the users and of the environment will be incorporated to make the interaction with the users more meaningful and to help resolve difficulties in interpreting natural language utterances of the users.

\section{Navigation assistance in the U-WISH project}

In the U-WISH (Usability of Web-based Information Services for Hypermedia) project (Neerincx et al., 1999) different support functions for web-based user interfaces are developed and tested with users. The project aims at developing a practical theory of web-navigation, and design guidelines for navigation support have been derived. A specific focus of the U-WISH project is the 'design for 
Van Dijk, Op den Akker, Nijholt, Zwiers

all' approach, aiming at the accessibility of web-based services for all types of users including elderly and disabled users.

To assist non-professional users the following navigation assistance functions have been added to the virtual theatre environment:

- A map of the environment can be asked for. That map complies with the advised map design principles described in the section about navigation assistance in general, so it is presented in the same orientation as the environment itself and the user's position is shown.

- Categorizing landmarks (different colors for different parts of the environment) have been added to the map, to support users to recognize in which part of the environment they are.

- A personal assistant, that has knowledge of the virtual environment and of the current user, is provided.

The U-WISH navigation experiments with the virtual theatre, compare navigation in the environment with this assistance and without assistance. User performance (effectiveness and efficiency) and satisfaction of visitors (young and old) will be compared. Here we only elaborate on the personal assistant and its characteristics.

The personal agent in the U-WISH environment is an unobtrusive advisor agent that helps the user to focus on relevant information for the current task and to find interesting places in the environment. Furthermore it can offer guidance when a user seems to 'walk in circles' or seems to miss parts of the environment. This agent is not embodied but takes the form of a narrow window that fits underneath the browser window, resembling a sort of toolbar. See figure 2 for a view at the hall of the virtual theatre with the personal agent that gives a suggestion. This figure also shows the rotating colored map.

This personal agent has knowledge of the virtual environment and of the current user (user profile and the user's present position and movement history). Because of this knowledge the assistant is able to dynamically provide user-specific advice to the individual visitor.

This agent is pro-active: it presents advice in the (small unobtrusive) window without being asked to do so by the user. In the future, it will also be possible to ask this agent to give advice or to make suggestions.

\section{Figure 2, hall of the virtual theatre with personal agent and colored map}

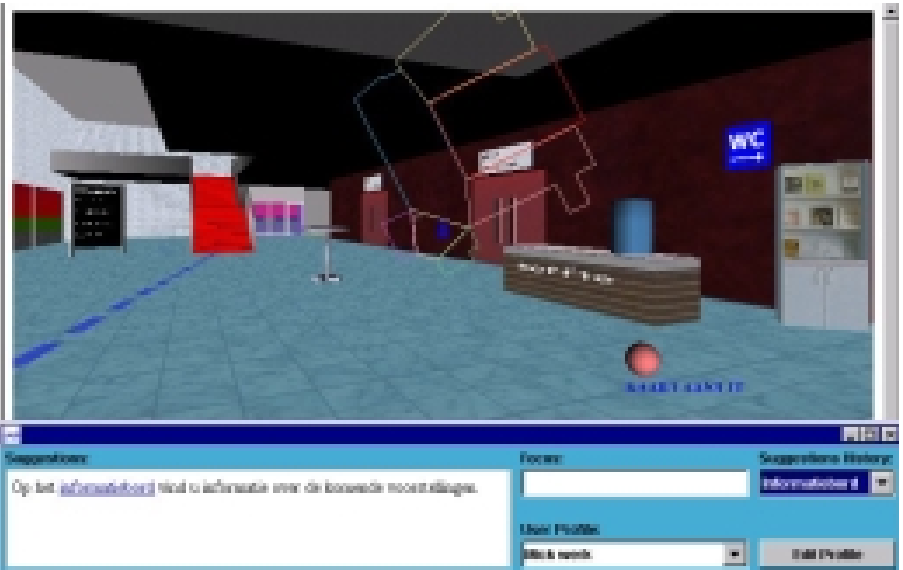

For experimental purposes the user profiles in the U-WISH project are currently fixed and put into a fill-in form by hand. These user profiles contain, among others, name and age of the user, profession, interests and transport constraints. The U-WISH agent is adaptive in the sense that the user's (changed) position is used to give advice. Adaptiveness in the sense of using dynamically changing user profiles is planned to be added in the near future.

\section{The navigation agent using natural language dialogue}

Another navigation agent we developed is concerned with geographical/physical wayfinding assistance in the virtual world. Using knowledge of the environment and of the user's present position in the virtual environment it guides users to a place they choose to go. This agent has knowledge about (or can compute):

- current position and focus of gaze of the user and what is in the eyesight of the visitor;

- objects, the properties they have, and geometric relations between objects and locations;

- possible paths towards objects and locations;

- the action it is currently performing (or has performed);

- previously visited locations or routes by the user;

- previous communications with the user.

The current environment offers the user some possibilities to interact with the navigation agent by means of a natural language dialogue. The visitor can choose between interaction modes, such as speech, keyboard, or mouse, or a combination of these. The natural language interaction between the navigation agent and the user allows the user to play an active role in the process of navigation. The navigation agent is reactive: the visitor can ask about existing locations in the theatre. The user can type a question like 


\section{Navigation Assistance in Virtual Worlds}

"where do I find the coffeebar?" or a command like "bring me to the coffeebar, please" and the system can react by answering the question in two ways: it can indicate the place on a map, or it can navigate the visitor's viewpoint through the environment along a route to this destination.

It will be clear that in order to do so the agent needs to know:

- how objects in the inventory of the environment are referred to by means of a natural language expression ('the coffeebar')

- how the actions it can perform can be referred to by means of natural language ('bring').

- what communicative act the user is performing by his utterance (is the user asking for information, or asking the system to do something)

Because the visitors will be aware of the visual context, in natural language interaction they will probably use references to that context. Hence natural language understanding cannot be seen as an isolated activity that is carried out by some language processing module that is independent of the virtual environment. Rather, the interpretation of natural language sentences is coupled to what is seen in the virtual world at the moment the sentence is uttered by the user. For instance, our advisor might suggest to go through 'the door', in case exactly one door is visible. The use of words like 'this', 'that', 'there', 'here' (deictic references) can only be understood by a natural language capable agent if this agent is able to recognize what is in the neighborhood of the user, or what can be seen by the user. Also the agent should be able to recognize objects that have recently been referred to in the dialogue and that could have been used in the utterance at that particular position. Such objects are stored in a focus list. We illustrate this by an example dialogue:

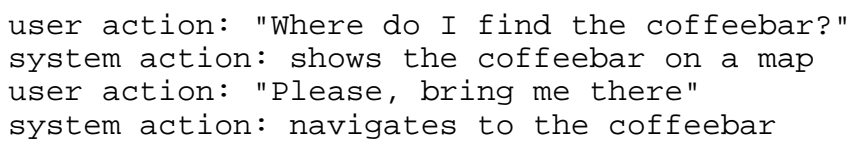

Since the system has been able to solve the coffeebar reference, and stored the information in the focus list, it can attach the indexical 'there' to the object referred to earlier in the dialogue. If the user asked the way to the coffeebar and then tries to find his way through the environment, the navigation agent should remember what the user is looking for so he can interrupt if he notices that the user navigates in a wrong direction: "you should go left here, if you look for the coffeebar". In case the reference problem could not be solved, the system can ask for more information. When the visitor's utterance is about performances, the navigation agent may attempt to contact Karin, the information and transaction agent.

The navigation agent is not embodied yet. Its viewpoint in the theatre is the current viewpoint from the position of the user. This agent is adaptive in the sense that it stores and uses information about the user's dialogue history, previously visited locations or routes and position, but it doesn't use a user profile.

\section{Conclusions and future work}

In this paper we discussed navigation assistance in virtual environments, and we gave an overview of the forms of navigation assistance in our virtual theatre that are currently being studied.

Various forms of navigation assistance are being developed. They all intend to support visitors, whether or not experienced, in finding their way in a virtual environment without previous training. Work in this area is often directed to particular user groups, e.g. elderly and disabled people. While navigation support is obviously relevant for these groups, it is probably more generally relevant. Geographically remote individuals may contact virtual worlds, and perhaps use it as a virtual place to meet. New interaction forms come within reach, in the areas of politics, commerce, and culture, and existing interaction forms may be strengthened as the threshold for first contacts is lowered.

In our research we emphasize personal agents, that is, assistants offering advice and suggestions that incorporate the most recent information available about the virtual environment. Personal agents, moreover, can be designed to offer assistance that complies with a visitor's user profile, they may or may not be embodied, and they may be proactive (acting without being asked by a user), reactive, or both. Obviously, personal agents can be very different in shaping, capacities, and functionality. Thus far we have created and studied three particular personal agents, as described above. Our future research will connect technical advancements, concerning agent theory and architectures, with human-computer interaction studies.

Regarding agent theory and architectures, one line of research will concern the improvement of agents' appearances in order to create 'believable agents' (Nijholt \& Hulstijn, 2000), which includes the aspects of embodiment, natural movement, gaze, facial expressions, and lip 


\section{Van Dijk, Op den Akker, Nijholt, Zwiers References}

movement synchronization. Research will also be conducted that seeks to enhance an agent's behavioral capacities by adding intelligence, increasing the use of (more extended) user profiles, and enhancing the capabilities to interpret spoken language and other non-discrete modalities of expression.

Studies in these areas all concentrate on single actors. An important further line of research will be the integration of various agents in a single virtual environment, which means that agents will have to be able to communicate and cooperate with one another while assisting users. Here, the question arises how a particular agent knows that it is being addressed in the situation that a user can direct $\mathrm{him} / \mathrm{herself}$ to several agents in the environment. And, in such a situation, how can an agent take part in a dialogue between the user and another agent?

Research in the area of human-computer interaction (HCI) will concern the contribution of various forms of assistance, personal agents in particular, to users' wayfinding behaviors and users' appreciation of the kind of assistance provided. The effects of separate design criteria (e.g. proactive or reactive) will be examined, as well as the impact of combined criteria. Objective effects will be examined (e.g. number of successful reservations made, pace of goalattainment in the context of geographical/physical wayfinding, number of users returning to the virtual theatre), as well as subjective effects (e.g. user satisfaction). The latter seems particularly important in cases that users visit the virtual theatre for exploration.

An important subject of research will concern the degree of changeability that is desirable or acceptable for users. As indicated, agents may become more adaptive, the virtual environment itself tends to become more complex (more landmarks, colours, maps), and in due course more agents may be present at the same time. The question is how users will respond to the abundance of stimuli that is present when several additions are combined. It is unlikely that all users will respond the same way. One of the main themes in HCI research is that different user categories can be distinguished, and that members from different categories need different amounts of assistance, different forms of assistance, and may have to be addressed in very dissimilar ways. This theme pertains to all subjects of study in HCI, not only the abundance of stimuli that is acceptable or useful.
Benyon, D. \& Höök, K. (1997). Navigation in information spaces: Supporting the individual. In: Howard, S., Hammond, J., \& Lindegaard, G., editors, Human-Computer Interaction: INTERACT'97, pp. 39-46. Chapman \& Hall.

Darken, R.P., \& Sibert, J.L. (1993). A toolset for navigation in virtual environments. In: Proceedings of UIST '93, Atlanta GA, pp. 157165. ACM.

Darken, R.P., \& Sibert, J.L. (1996). Wayfinding strategies and behaviors in large virtual worlds. In: Proceedings of CHI '96, pp. 142-149. ACM.

Krieg-Brückner, B., Röfer, T., Carmesin, H.O., \& Müller, R. (1998). A taxonomie of spatial knowledge for navigation and its application to the Bremen autonomous wheelchair. In: Freksa, C., Habel, C., \& Wender, K.F., editors, Spatial Cognition, Lecture Notes in Artificial Intelligence 1404, pp. 373-397. Berlin: Springer Verlag.

Nash, E.B., Edwards, G.W., Thompson, J.A., \& Barfield, W. (2000). A review of presence and performance in virtual environments. International Journal of Human-Computer Interaction, 12, pp. 1-41.

Neerincx, M.A., \& Lindenberg, J. (1999). Supporting individual situation awareness in web-environments. In: Pikaar, E., editor, Ergonomie in Uitvoering: De Digitale Mens, pp. 144-155. Nederlandse Vereniging voor Ergonomie.

Neerincx, M.A., Lindenberg, J., Rypkema, J., \& Van Besouw, S. (2000). A practical cognitive theory of web-navigation: Explaining agerelated performance differences. In: Position Paper CHI 2000 Workshop Basic Research Symposium. ACM.

M.A. Neerincx, S. Pemberton and J. Lindenberg (1999). U-WISH; Web usability: methods, guidelines and support interfaces. TNO-report TM-99-D005, TNO Human Factors Research Institute.

Nijholt (2000a). Virtual malls for commerce and entertainment. In: Linnhof-Popien, C., \& Hegering, H.G., editors, Proceedings 3 rd IFIP/GI International Conference on Trends towards a Universal Services Market (USM 2000), LNCS 1890, pp. 326-333, Muenchen.

Nijholt (2000b). Towards virtual communities on the Web: Actors and audience. In: Proceedings ICSC Symposium on Interactive and Collaborative Computing (ICC'2000). University of Wollongong, Australia, December 2000, to appear.

Nijholt, A.. \& Hulstijn, J. (2000). Multimodal interactions with agents in virtual worlds. In Kasabov, N. (ed.), Future directions for Intelligent Information Systems and Information Science, pp. 148-173. Physica-Verlag: Studies in Fuzziness and Soft Computing.

Satalich, G. (1995). Navigation and wayfinding in virtual reality: Finding proper tools and cues to enhance navigational awareness. Master's thesis, University of Washington. 


\section{Navigation Assistance in Virtual Worlds}

Spence, R. (1999). A framework for navigation. International Journal of Human-Computer Studies, 51, pp. 919-945.

Vinson, N. (1999). Design guidelines for landmarks to support navigation in virtual environments. In: Proceedings of CHI '99, pp. 278285. ACM.

Volbracht, V., \& Domik, G. (2000). Developing effective navigation techniques in virtual 3d environments. In: Mulder, J.D., \& Van Liere, R., editors, Virtual Environments 2000: Proceedings of the Eurographics Workshop 2000, pp. 55-64. Springer.

Werner, S., Krieg-Brückner, B., \& Herrmann, T. (2000). Modelling navigational knowledge by route graphs. In: Freksa, C., Brauer, W., \& Wender, K.F., editors, Spatial Cognition II, Lecture Notes in Artificial Intelligence 1849, pp. 295-316. Berlin: Springer Verlag.

\section{Biographies}

Betsy van Dijk is assistant professor in human-computer interaction with special interest in usability issues.

Rieks op den Akker is assistant professor in natural language processing.

Anton Nijholt is full professor of Computer Science and currently interested in interaction modeling in virtual environments.

Job Zwiers is associate professor in Computer Science, working on formal methods applied to agent-based systems. 